MATHEMATICS OF COMPUTATION

Volume 70 , Number 235 , Pages $935-949$

S $0025-5718(00) 01244-8$

Article electronically published on March 1, 2000

\title{
AN ITERATIVE SUBSTRUCTURING METHOD FOR MAXWELL'S EQUATIONS IN TWO DIMENSIONS
}

\author{
ANDREA TOSELLI, OLOF B. WIDLUND, AND BARBARA I. WOHLMUTH
}

\begin{abstract}
Iterative substructuring methods, also known as Schur complement methods, form an important family of domain decomposition algorithms. They are preconditioned conjugate gradient methods where solvers on local subregions and a solver on a coarse mesh are used to construct the preconditioner. For conforming finite element approximations of $H^{1}$, it is known that the number of conjugate gradient steps required to reduce the residual norm by a fixed factor is independent of the number of substructures, and that it grows only as the logarithm of the dimension of the local problem associated with an individual substructure. In this paper, the same result is established for similar iterative methods for low-order Nédélec finite elements, which approximate $H(\operatorname{curl} ; \Omega)$ in two dimensions. Results of numerical experiments are also provided.
\end{abstract}

\section{INTRODUCTION}

In this paper, we consider the following boundary value problem

$$
\begin{aligned}
L \mathbf{u}:=\operatorname{curl}(a \operatorname{curl} \mathbf{u})+B \mathbf{u} & =\mathbf{f} \text { in } \Omega, \\
\mathbf{n} \times \mathbf{u} & =0 \text { on } \partial \Omega .
\end{aligned}
$$

Here $\Omega$ is a bounded polygonal domain in $\mathbb{R}^{2}$ of unit diameter, and $\mathbf{n}$ its outward normal. We assume that $\mathbf{f} \in\left(L^{2}(\Omega)\right)^{2}$, that the coefficient matrix $B$ is a symmetric uniformly positive matrix-valued function with $b_{i, j} \in L^{\infty}(\Omega), 1 \leq i, j \leq 2$, and that $a \in L^{\infty}(\Omega)$ is a positive function bounded away from zero. The unit tangential vector $\mathbf{t}$ on $\partial \Omega$ is defined such that, following its direction, $\partial \Omega$ is traversed counterclockwise. The tangential component of $\mathbf{u}$ on $\partial \Omega, \mathbf{u} \cdot \mathbf{t}$, is then equal to $\mathbf{n} \times \mathbf{u}$.

Received by the editor August 14, 1998 and, in revised form, September 7, 1999.

2000 Mathematics Subject Classification. Primary 65N30, 65N55, 65F10, 78M10.

Key words and phrases. Maxwell's equations, Nédélec finite elements, domain decomposition, iterative substructuring methods.

The work of the first author was supported in part by the National Science Foundation under Grants NSF-CCR-9732208 and NSF-ECS-9527169, and in part by the U.S. Department of Energy under Contract DE-FG02-92ER25127.

The work of the second author was supported in part by the National Science Foundation under Grants NSF-CCR-9732208 and NSF-ECS-9527169, and in part by the U.S. Department of Energy under Contract DE-FG02-92ER25127.

The work of the third author was supported in part by the Deutsche Forschungsgemeinschaft. 
The choice of Dirichlet boundary conditions is made for simplicity only; other boundary conditions may also be considered without any new technical complications. We could, in particular, consider reflection conditions of Neumann or Robin type, which are related to Silver-Müller radiation conditions; see [16].

Equation (1) is encountered when solving Maxwell's equations and Stokes' problem in the stream function-vorticity formulation; see [8, 10]. The case of timedependent Maxwell's equations, discretized with an implicit time-scheme, is particularly important; in this case, $\mathbf{u}$ is the electric field and equation (1) is solved in each time step. The coefficient $a$ vanishes with the time step, and $\mathbf{f}$ depends on the solution at the previous steps, as well as the electric current density; see [8, 15].

The spaces $H(\operatorname{curl} ; \Omega)$ and $H(\operatorname{div} ; \Omega)$, and special finite element approximations have been introduced to analyze equations such as (1); see [10. In recent years, a considerable effort has been made to develop optimal or quasi-optimal, scalable preconditioners for these finite element approximations of problems in $H(\operatorname{curl} ; \Omega)$ and $H(\operatorname{div} ; \Omega)$. Two-level overlapping Schwarz preconditioners for these spaces have been developed for two (see [3]) and three (see [20, 13]) dimensions, respectively. Multigrid and multilevel methods are considered in [3, 5, 12, 11, 13, 4]. Since this paper was submitted for publication, several further results have been obtained (see [25, 19, 22, 21]). We know of no previous work on iterative substructuring methods for $H(\operatorname{div} ; \Omega)$, and only a few papers on the $H(\operatorname{curl} ; \Omega)$ case (see 2 ]) where optimality is proven for a two-subdomain iterative substructuring preconditioner, combined with Richardson's method, for a low-frequency approximation of timeharmonic Maxwell's equations in three dimensions.

Iterative substructuring algorithms are iterative methods, where the preconditioner is built from solvers defined on the substructures which form a nonoverlapping partition of the original domain. When a coarse solver is added, the rate of convergence can be made independent of the number of subregions. The method considered here has its roots in the early work by Bramble, Pasciak, and Schatz [6]; see also [23. That work is all for the $H^{1}$ case. There has been extensive work on the three dimensional case as well; see, e.g., Dryja, Smith, and Widlund [9] and the many references therein. We note that for all these iterative substructuring methods, the condition number of the relevant iteration operator grows polylogarithmically in $H / h$. Here $H$ represents the diameter of a substructure, and $h$ the diameter of the elements into which the substructure has been divided. The bounds are independent of the number of subregions and also of possible jumps in the coefficients across the interface between the substructures.

In this paper, we restrict ourselves to two dimensions and develop an iterative substructuring method for equation (1). The condition number bound and the performance are very similar to those previously known for the $H^{1}$ case. The bounds are independent of the number of substructures; they are developed locally for one substructure at a time and they are therefore insensitive to even large changes in the coefficients from one substructure to its neighbors. We will also discuss the impact on the performance when the coefficients $a$ and $B$ change relative to each other; see Sections 4 and 5 .

This paper is organized as follows. In Section 2, we recall some properties of the space $H(\operatorname{curl} ; \Omega)$ and introduce a variational formulation of (1). In Section 3, we describe the finite element spaces employed for the approximation of (1) and prove a stability lemma for an interpolation operator. We describe our substructuring 
preconditioner and prove an upper bound for its condition number in Section 4 . The last section is devoted to the discussion of some numerical results.

\section{Problem Setting And functional SPaCes}

Given a bounded open Lipschitz domain $\mathcal{D} \subset \mathbb{R}^{2}$, let $(\cdot, \cdot)_{s ; \mathcal{D}}$ denote the scalar product in the Sobolev space $H^{s}(\mathcal{D})$. We use $\|\cdot\|_{s ; \mathcal{D}}$ and $|\cdot|_{s ; \mathcal{D}}$ to denote the corresponding norm and semi-norm, respectively, dropping the subscript $\mathcal{D}$ if $\mathcal{D}=$ $\Omega$. For a general reference on Sobolev spaces, see [1].

The weak formulation of problem (1) is defined in

$$
H(\operatorname{curl} ; \Omega):=\left\{\mathbf{v} \in\left(L^{2}(\Omega)\right)^{2} \mid \quad \operatorname{curl} \mathbf{v} \in L^{2}(\Omega)\right\} .
$$

This is a Hilbert space with the inner product and the associated graph norm defined by

$$
(\mathbf{u}, \mathbf{v})_{\text {curl }}:=(\mathbf{u}, \mathbf{v})_{0}+(\operatorname{curl} \mathbf{u}, \operatorname{curl} \mathbf{v})_{0}, \quad\|u\|_{\text {curl }}^{2}:=(\mathbf{u}, \mathbf{u})_{0}+(\operatorname{curl} \mathbf{u}, \operatorname{curl} \mathbf{u})_{0} ;
$$

see 10] for a discussion of basic properties of $H(\operatorname{curl} ; \Omega)$. In particular, we recall a trace theorem:

Given a vector $\mathbf{u} \in H(\operatorname{curl} ; \Omega)$, then its tangential component on the boundary, $\mathbf{n} \times \mathbf{u}=\mathbf{u} \cdot \mathbf{t}$, belongs to the space $H^{-\frac{1}{2}}(\partial \Omega)$. The subspace of vectors in $H(\operatorname{curl} ; \Omega)$ with vanishing tangential component on $\partial \Omega$ is denoted by $H_{0}(\operatorname{curl} ; \Omega)$.

Equation (1) can be given the following variational form.

Find $\mathbf{u} \in H_{0}(\operatorname{curl} ; \Omega)$ such that

$$
a(\mathbf{u}, \mathbf{v})=(\mathbf{f}, \mathbf{v})_{0}, \quad \mathbf{v} \in H_{0}(\operatorname{curl} ; \Omega),
$$

where the bilinear form $a(\cdot, \cdot)$ is given by

$$
a(\mathbf{u}, \mathbf{v}):=\int_{\Omega}(a \operatorname{curl} \mathbf{u} \operatorname{curl} \mathbf{v}+B \mathbf{u} \cdot \mathbf{v}) d x, \quad \mathbf{u}, \mathbf{v} \in H(\operatorname{curl} ; \Omega) .
$$

Associated with the bilinear form $a(\cdot, \cdot)$ is the energy norm $\|\cdot\|_{a}$ defined by $\|\mathbf{v}\|_{a}^{2}:=$ $a(\mathbf{v}, \mathbf{v})$. Our assumptions on the coefficients guarantee that the energy norm is equivalent to the graph norm.

A central result, valid for any Lipschitz domain, is a Helmholtz type decomposition of $H_{0}(\operatorname{curl} ; \Omega)$ which is orthogonal with respect to the graph norm (see [8, vol. 3 , Proposition 1, p. 215]):

$$
H_{0}(\operatorname{curl} ; \Omega)=\operatorname{grad} H_{0}^{1}(\Omega) \oplus H_{0}^{\perp}(\operatorname{curl} ; \Omega) .
$$

Here

$$
H_{0}^{\perp}(\operatorname{curl} ; \Omega):=H^{0}(\operatorname{div} ; \Omega) \cap H_{0}(\operatorname{curl} ; \Omega)=\operatorname{curl} H^{1}(\Omega) \cap H_{0}(\operatorname{curl} ; \Omega),
$$

and

$$
H^{0}(\operatorname{div} ; \Omega):=\{\mathbf{u} \in H(\operatorname{div} ; \Omega) \mid \operatorname{div} \mathbf{u}=0\},
$$

where $H(\operatorname{div} ; \Omega):=\left\{\mathbf{u} \in\left(L^{2}(\Omega)\right)^{2} \mid \operatorname{div} \mathbf{u} \in L^{2}(\Omega)\right\}$.

If $\partial \Omega$ is connected, the kernel of the curl operator in $H_{0}(\operatorname{curl} ; \Omega)$ is $\operatorname{grad} H_{0}^{1}(\Omega)$ (see [8, vol. 3, Proposition 2, p. 219]), and the inequality

$$
\|\mathbf{u}\|_{0} \leq C \operatorname{diam}(\Omega)\|\operatorname{curl} \mathbf{u}\|_{0}, \mathbf{u} \in H_{0}^{\perp}(\operatorname{curl} ; \Omega)
$$

holds, with a constant $C$ independent of $\mathbf{u}$. 


\section{NÉDÉLEC FINITE ELEMENT DISCRETIZATIONS}

We first introduce a simplicial triangulation $\mathcal{T}_{H}$ and then a family of quasiuniform and shape-regular triangulations $\mathcal{T}_{h}, h<H$, obtained by refining $\mathcal{T}_{H}$ in some standard way. Let $\mathcal{T}_{H}=\left\{T_{k}, k=1, \ldots, K\right\}$ and $H_{k}=\operatorname{diam}\left(T_{k}\right)$, with $H=\max \left\{H_{k}\right\}$. The coarse elements $T_{k}$ are also called substructures. We denote the set of edges associated with the triangulations $\mathcal{T}_{H}$ and $\mathcal{T}_{h}$, by $\mathcal{E}_{H}$ and $\mathcal{E}_{h}$, respectively. We consider, in full detail, only triangulations based on triangles but note that the results of this paper are equally valid for finite element spaces built on quadrilaterals.

We assume that the coefficients $a$ and $B$ in equation (11) are constant in each substructure $T_{k} \in \mathcal{T}_{H}$ and denote them by $a_{k}$ and $B_{k}$, respectively. We also assume that

$$
0<\beta_{k}|\mathbf{x}|^{2} \leq \mathbf{x}^{T} B_{k} \mathbf{x} \leq \gamma_{k}|\mathbf{x}|^{2}, \quad \mathbf{x} \in \mathbb{R}^{2}
$$

for $k=1, \ldots, K$.

We consider finite element discretizations based on the lowest order Nédélec elements, also known as Whitney finite elements; see [7, 10, 14. With $\mathbb{P}_{\ell}(D)$, $D \subseteq \Omega, \ell \geq 0$, the set of polynomials of degree $\leq \ell$ on $\mathrm{D}$, and with

$$
\mathcal{R}(T):=\mathbb{P}_{0}(T)^{2}+\mathbb{P}_{0}(T)\left(x_{2},-x_{1}\right)^{T},
$$

for $T$ a triangle, we define the following spaces:

$$
X_{H}(\Omega):=\left\{\mathbf{u} \in H(\operatorname{curl} ; \Omega) \mid \quad \mathbf{u}_{\left.\right|_{T}} \in \mathcal{R}(T), T \in \mathcal{T}_{H}\right\}
$$

and

$$
X_{h}(\Omega):=\left\{\mathbf{u} \in H(\operatorname{curl} ; \Omega) \mid \quad \mathbf{u}_{\left.\right|_{T}} \in \mathcal{R}(T), T \in \mathcal{T}_{h}\right\} .
$$

The degrees of freedom are given by averages over the edges $e$ of the triangulations

$$
\lambda_{e}(\mathbf{u}):=\frac{1}{h_{e}} \int_{e} \mathbf{n} \times \mathbf{u} d \sigma,
$$

where $h_{e}$ is the length of the edge $e$.

Subspaces of vectors with a vanishing tangential component are defined by

$$
X_{0 ; h}(\Omega):=X_{h}(\Omega) \cap H_{0}(\operatorname{curl} ; \Omega), \quad X_{0 ; H}(\Omega):=X_{H}(\Omega) \cap H_{0}(\operatorname{curl} ; \Omega) .
$$

As in the case of Lagrangian finite elements the $L^{2}$-norm of the Nédélec elements can be bounded from above and below by means of the values of their degrees of freedom. The proof in [17, Proposition 6.3.1] for Lagrangian elements can easily be adapted to establish the following lemma.

Lemma 3.1. There exist constants $0<c_{1} \leq C_{1}$, which depend only on the minimal angle of the elements in $\mathcal{T}_{h},\left(\mathcal{T}_{H}\right)$, such that for all $\mathbf{u} \in \mathcal{R}(T), T \in \mathcal{T}_{h},\left(\mathcal{T}_{H}\right)$,

$$
c_{1} \sum_{e \subset \partial T}\left(h_{e} \lambda_{e}(\mathbf{u})\right)^{2} \leq\|\mathbf{u}\|_{0 ; T}^{2} \leq C_{1} \sum_{e \subset \partial T}\left(h_{e} \lambda_{e}(\mathbf{u})\right)^{2} .
$$

An essential tool in our proof for the Schwarz methods is an interpolation operator $\rho_{H}$ onto $X_{H}(\Omega)$, defined in terms of the degrees of freedom of $X_{H}(\Omega)$, i.e.,

$$
\lambda_{e}\left(\rho_{H} \mathbf{u}\right):=\frac{1}{h_{e}} \int_{e} \mathbf{n} \times \mathbf{u} d \sigma, \quad e \in \mathcal{E}_{H} .
$$


We note that $\lambda_{e}\left(\rho_{H} \mathbf{u}\right)$ depends solely on the value of $\mathbf{u}$ on the coarse edge $e$; this will allow us to develop our bounds locally, one subregion at a time.

It is easy to show that $\operatorname{curl}\left(\rho_{H} \mathbf{u}\right)=\Pi_{H} \operatorname{curl}(\mathbf{u})$, where $\Pi_{H}$ is the $L^{2}$-projection onto the space of piecewise constant functions associated with the triangulation $\mathcal{T}_{H}$, since for each $T \in \mathcal{T}_{H}$

$$
\begin{aligned}
\left.\left(\operatorname{curl}\left(\rho_{H} \mathbf{u}\right)\right)\right|_{T} & =\frac{1}{|T|} \int_{T} \operatorname{curl}\left(\rho_{H} \mathbf{u}\right) d x=\frac{1}{|T|} \int_{\partial T}\left(\rho_{H} \mathbf{u}\right) \times \mathbf{n} d \sigma \\
& =\frac{1}{|T|} \int_{\partial T} \mathbf{u} \times \mathbf{n} d \sigma=\frac{1}{|T|} \int_{T} \operatorname{curl}(\mathbf{u}) d x=\left.\left(\Pi_{H} \operatorname{curl}(\mathbf{u})\right)\right|_{T} .
\end{aligned}
$$

The following lemma establishes the stability of the interpolant $\rho_{H}$.

Lemma 3.2. There exists a constant $C>0$, which depends only on the minimal angle of the triangulations $\mathcal{T}_{h}$ and $\mathcal{T}_{H}$, such that for all $\mathbf{u} \in X_{h}(\Omega)$

$$
\begin{aligned}
\left\|\operatorname{curl}\left(\rho_{H} \mathbf{u}\right)\right\|_{0}^{2} & \leq\|\operatorname{curl} \mathbf{u}\|_{0}^{2}, \\
\left\|\rho_{H} \mathbf{u}\right\|_{0}^{2} & \leq C\left(\left(1+\log \left(\frac{H}{h}\right)\right)\|\mathbf{u}\|_{0}^{2}+H^{2}\|\operatorname{curl} \mathbf{u}\|_{0}^{2}\right) .
\end{aligned}
$$

Proof. Inequality (10) is obtained by using (9) and summing over the contributions from the different substructures.

The proof of (111) uses arguments similar to those of [24] in which a multilevel splitting of the div-operator was considered. We consider one subdomain at a time. Given $T \in \mathcal{T}_{H}$, let $e$ be one of its edges of length $H_{e}$; we use this notation here to distinguish its length from those of the edges of the fine triangulation. Let $v_{1}$ and $v_{2}$ be the endpoints of $e$. The restriction of the fine triangulation $\mathcal{T}_{h}$ to $e$ splits $e$ into a union of nonoverlapping edges of the fine triangulation. Let $e_{1}$ and $e_{2}$ be the edges, which end at $v_{1}$ and $v_{2}$, respectively, and let $t_{1}$ and $t_{2}$ be the elements in $\mathcal{T}_{h}$ to which $e_{1}$ and $e_{2}$ belong. We now define a continuous, piecewise linear function $\vartheta_{e}$ on $\partial T$, which is equal to one on $e$, except on $e_{1}$ and $e_{2}$, where it decreases linearly to zero; it is extended by zero on $\partial T \backslash e$. As shown in [18, Section 5.3.2], $\vartheta_{e}$ can be extended to $T$, as a continuous piecewise linear function, still denoted by $\vartheta_{e}$, with an absolute value less than or equal to 1 , and with a gradient which is bounded by $C / h$ on $t_{1}$ and $t_{2}$ and by $C / r$ elsewhere. Here $r$ is the distance to the closest of $v_{1}$ or $v_{2}$.

Because of Lemma 3.1, it is enough to bound $H_{e}\left(\mathbf{n} \times \rho_{H} \mathbf{u}\right)_{\left.\right|_{e}}$, for each edge $e \subset \partial T$. Since the function $\mathbf{n} \times\left(\rho_{H} \mathbf{u}\right)_{\mid}$is constant, we can use Stokes' theorem, (77) and (8), and find that

$$
\begin{aligned}
& H_{e}\left(\mathbf{n} \times \rho_{H} \mathbf{u}\right)_{\left.\right|_{e}}=\int_{e}(\mathbf{n} \times \mathbf{u}) d \sigma \\
& =\int_{\partial T_{k}} \vartheta_{e}(\mathbf{n} \times \mathbf{u}) d \sigma+\frac{h_{e_{1}}}{2}\left(\mathbf{n} \times \mathbf{u}_{\left.\right|_{e_{1}}}\right)+\frac{h_{e_{2}}}{2}\left(\mathbf{n} \times \mathbf{u}_{\left.\right|_{e_{2}}}\right) \\
& =\int_{T_{k}}\left(\vartheta_{e} \operatorname{curl} \mathbf{u}+\operatorname{grad} \vartheta_{e} \times \mathbf{u}\right) d x+\frac{h_{e_{1}}}{2}\left(\mathbf{n} \times \mathbf{u}_{\left.\right|_{e_{1}}}\right)+\frac{h_{e_{2}}}{2}\left(\mathbf{n} \times \mathbf{u}_{\left.\right|_{e_{2}}}\right) .
\end{aligned}
$$

Thus, the absolute value of $H_{e}\left(\mathbf{n} \times \rho_{H} \mathbf{u}\right)_{\left.\right|_{e}}$ can be bounded from above by

$$
C\left(H\|\operatorname{curl} \mathbf{u}\|_{0 ; T}+\left|\vartheta_{e}\right|_{1 ; T}\|\mathbf{u}\|_{0 ; T}+\|\mathbf{u}\|_{0 ; t_{1}}+\|\mathbf{u}\|_{0 ; t_{2}}\right) .
$$


We next consider the second term on the right-hand side of (12) in more detail. To obtain an upper bound for $\left|\vartheta_{e}\right|_{1 ; T}$, we split $T$ into $t_{1} \cup t_{2}$ and $T \backslash\left(t_{1} \cup t_{2}\right)$,

$$
\begin{aligned}
\left|\vartheta_{e}\right|_{1 ; T}^{2} & =\int_{t_{1} \cup t_{2}}\left|\operatorname{grad} \vartheta_{e}\right|^{2} d x+\int_{T \backslash\left(t_{1} \cup t_{2}\right)}\left|\operatorname{grad} \vartheta_{e}\right|^{2} d x \\
& \leq C\left(\int_{t_{1} \cup t_{2}} \frac{1}{h^{2}} d x+\int_{T \backslash\left(t_{1} \cup t_{2}\right)} \frac{1}{r^{2}} d x\right) \leq C\left(1+\int_{h} \int_{0}^{2 \pi} \frac{1}{r} d \phi d r\right) \\
& \leq C\left(1+\log \left(\frac{H}{h}\right)\right) .
\end{aligned}
$$

Taking ([8), (12), and (13) into account, we find, by summing over all $e \subset \partial T$, that

$$
\left\|\rho_{H} \mathbf{u}\right\|_{0 ; T}^{2} \leq H^{2}\|\operatorname{curl} \mathbf{u}\|_{0 ; T}^{2}+C\left(1+\log \left(\frac{H}{h}\right)\right)\|\mathbf{u}\|_{0 ; T}^{2}
$$

The final result is obtained by summing over all $T \in \mathcal{T}_{H}$.

Remark 3.3. We can obtain a similar estimate for the energy norm. However in that case, the constant also depends on the ratio of the coefficients. We find,

$$
\begin{aligned}
\int_{\Omega} B\left(\rho_{H} \mathbf{u}\right) \cdot\left(\rho_{H} \mathbf{u}\right) d x \leq & C \max _{1 \leq k \leq K} \frac{\gamma_{k}}{\beta_{k}}\left(1+\log \left(\frac{H}{h}\right)\right) \int_{\Omega} B \mathbf{u} \cdot \mathbf{u} d x \\
& +C \max _{1 \leq k \leq K} \frac{H_{k}^{2} \gamma_{k}}{a_{k}} \int_{\Omega} a \operatorname{curl} \mathbf{u} \operatorname{curl} \mathbf{u} d x .
\end{aligned}
$$

We will need an orthogonal splitting for the discrete spaces, similar to that of (3). We refer to [10, 7, 3] for details and note that the results given in [7, Section III.3], [7, Section IV.1], and [3] for the $H(\operatorname{div} ; \Omega)$ case are also valid for $H(\operatorname{curl} ; \Omega)$, since the vectors in $H(\operatorname{curl} ; \Omega)$ can be obtained from those in $H(\operatorname{div} ; \Omega)$ by a 90 -degree rotation. Let $T \in \mathcal{T}_{H}$ be an element of the coarse triangulation and let $X_{0 ; h}(T)$ be the finite element space $H_{0}(\operatorname{curl} ; T) \cap X_{h}$, defined on $T$ and with vanishing tangential component. Furthermore, let $S_{0 ; h}(T)$ denote the space of functions which are constant on each element of $\mathcal{T}_{h}$ and with mean value zero on $T$, and let $W_{0 ; h}(T)$ be the space of continuous functions which are linear on each element in $\mathcal{T}_{h}$ and vanish on $\partial T$.

It is well known (see [7, 10]) that, since $\partial T$ is connected,

$$
\left\{\mathbf{u} \in X_{0 ; h}(T) \mid \operatorname{curl} \mathbf{u}=0\right\}=\left\{\operatorname{grad} p \mid p \in W_{0 ; h}(T)\right\},
$$

and that

$$
\operatorname{curl} X_{0 ; h}(T)=S_{0 ; h}(T) .
$$

We define the following orthogonal decomposition

$$
X_{0 ; h}(T)=\operatorname{grad} W_{0 ; h}(T) \oplus X_{0 ; h}^{\perp}(T),
$$

where $X_{0 ; h}^{\perp}(T)$ is defined by

$$
X_{0 ; h}^{\perp}(T):=\left\{\mathbf{v} \in X_{0 ; h}(T) \mid(\mathbf{v}, \mathbf{w})_{\text {curl }}=0, \mathbf{w} \in \operatorname{grad} W_{0 ; h}(T)\right\} .
$$


The pair of subspaces $X_{0 ; h}(T)$ and $S_{0 ; h}(T)$ also satisfies a Babuška-Brezzi condition (see [7])

$$
\inf _{\substack{p \in S_{0 ; h}(T) \\ p \neq 0}} \sup _{\substack{\mathbf{u} \in X_{0 ; h}(T) \\ \mathbf{u} \neq 0}} \frac{(p, \operatorname{curl} \mathbf{u})_{0}}{\|\mathbf{u}\|_{\text {curl; }}\|p\|_{0 ; T}} \geq c>0 .
$$

The constant $c$ is independent of $h$ but depends on the shape of $T$. An immediate consequence of (16) is that for each $\mathbf{u} \in X_{0 ; h}(T)$, there is a unique $\mathbf{v} \in X_{0 ; h}^{\perp}(T)$ with $\operatorname{curl} \mathbf{v}=\operatorname{curl} \mathbf{u}$ such that

$$
\|\mathbf{v}\|_{0 ; T} \leq C H\|\operatorname{curl} \mathbf{v}\|_{0 ; T}
$$

with a constant independent of v; see [10, Proposition 5.1].

\section{SchWARZ Methods AND Stable SPLitTings}

Schwarz theory provides powerful tools for the study of many classes of preconditioners for partial differential equations; see, e.g., [18. Applications are particularly well developed for conforming finite element approximations of elliptic problems. We recall that a Schwarz algorithm is an iteration scheme defined in terms of a family of subspaces $\left\{V_{i}, i=0, \ldots, J\right\}$, projection-like operators onto these subspaces, and a scalar product on a relevant finite dimensional space $V$. Here, we restrict ourselves to using exact orthogonal projections $P_{i}$ onto the subspaces $V_{i}$ with respect to the bilinear form $a(\cdot, \cdot)$. An additive Schwarz method provides a new operator equation, with the same solution as the given finite element problem,

$$
P_{a s} \mathbf{u}=\sum P_{i} \mathbf{u}=\mathbf{g},
$$

with an operator which can be much better conditioned than that of the original discrete elliptic problem; it can often be solved effectively by the conjugate gradient method, without further preconditioning, employing $a(\cdot, \cdot)$ as the inner product. The right-hand side $\mathbf{g}$ can be chosen so that the new problem has the same solution as the original one; it is possible to compute $P_{i} \mathbf{u}$ from the data given by the original problem.

An estimate for the lowest eigenvalue of $P_{a s}$ is given by the following lemma; see [18] Secton 5.2].

Lemma 4.1. If a representation, $\mathbf{u}=\sum \mathbf{u}_{i}, \mathbf{u}_{i} \in V_{i}$, can be found such that

$$
\sum a\left(\mathbf{u}_{i}, \mathbf{u}_{i}\right) \leq C_{0}^{2} a(\mathbf{u}, \mathbf{u}) \quad \forall \mathbf{u} \in V
$$

then the lowest eigenvalue of the additive Schwarz operator $P_{a s}$ is bounded from below by $C_{0}^{-2}$.

In the case at hand, we proceed by first introducing an auxiliary decomposition of the Nédélec space $X_{0 ; h}(\Omega)$, related to the Laplace operator, and prove, in Lemma 4.2, that a stable splitting can be found. We then use this result to prove a lower bound for the smallest eigenvalue of an additive iterative substructuring method. We conclude this section by showing that there is also a bound that is independent of the the ratio of the coefficients $B$ and $a$ in (11). We also note that, as is often the case, a good bound for the largest eigenvalue is routine and can be obtained by a standard coloring argument; see, e.g., [18, Page 165]. In particular, the coefficients $a$ and $B$ do not enter into the upper bound. We only consider in our analysis decompositions of the whole space $X_{0 ; h}$, but note that the method presented here can also be employed to define a preconditioner for the 
Schur complement system, obtained by eliminating the variables in the interior of the substructures with a direct method. We refer to [18 and to the references therein, for a discussion of some practical issues of Schur complement methods.

We begin by introducing a Schwarz method based on a direct sum decomposition of the finite element space. An edge in $\mathcal{T}_{H}$ is denoted by $\Gamma_{i j}$ if it is shared by the substructures $T_{i}$ and $T_{j}$. We also define a region $T_{i j}$ by $\bar{T}_{i j}:=\overline{T_{i} \cup T_{j}}$.

We first consider the following splitting of $X:=X_{0 ; h}(\Omega)$ into subspaces:

$$
X=X_{0}+\sum_{k=1}^{K} X_{k}+\sum_{\substack{i, j=1 \\ i<j}}^{K} X_{i j}
$$

Here $X_{0}:=X_{0 ; H}(\Omega)$ is the finite element space on the coarse triangulation $\mathcal{T}_{H}$. $X_{k}$ is the subspace of vectors with support in $\bar{T}_{k}: X_{k}:=\left\{\mathbf{v} \in X|\mathbf{v}|_{\Omega \backslash \bar{T}_{k}}=0\right\}$. Finally, the space $X_{i j}$ consists of the gradient of functions in $W_{0 ; h}\left(T_{i j}\right)$, which are discrete harmonic with respect to the Laplace operator on $T_{i}$ and $T_{j}$, i.e., they are the extensions with the smallest $H^{1}$ semi-norm of all finite element functions with the given boundary values.

It is then easy to see that

$$
X_{0} \cap X_{k}=X_{0} \cap X_{i j}=X_{i j} \cap X_{k}=\{0\}, \quad 1 \leq k \leq K, \quad 1 \leq i<j \leq K .
$$

Furthermore, the space $X_{k}$ and $X_{l}$ as well as $X_{i j}$ and $X_{n m}$ have an empty intersection for different sets of indices. Counting the degrees of freedom then guarantees that $(18)$ is a direct sum.

We note that the elements of $X_{i j}$ are not defined by solving a homogeneous Maxwell equation with boundary data given by piecewise constant functions, with zero averages over the edges; see below for a discussion of that case.

Lemma 4.2. For each $\mathbf{u} \in X$, there exists a unique decomposition

$$
\mathbf{u}=\mathbf{u}_{0}+\sum_{k=1}^{K} \mathbf{u}_{k}+\sum_{\substack{i, j=1 \\ i<j}}^{K} \mathbf{u}_{i j}
$$

$\mathbf{u}_{0} \in X_{0}, \mathbf{u}_{k} \in X_{k}, \mathbf{u}_{i j} \in X_{i j}$, such that

$$
a\left(\mathbf{u}_{0}, \mathbf{u}_{0}\right)+\sum_{k=1}^{K} a\left(\mathbf{u}_{k}, \mathbf{u}_{k}\right)+\sum_{\substack{i, j=1 \\ i<j}}^{K} a\left(\mathbf{u}_{i j}, \mathbf{u}_{i j}\right) \leq C\left(1+\log \left(\frac{H}{h}\right)\right)^{2} a(\mathbf{u}, \mathbf{u})
$$

with a constant $C>0$, independent of $h, H$, and $\mathbf{u}$.

Proof. We find that $\mathbf{u}_{0}=\rho_{H} \mathbf{u}$ since the decomposition is unique and $\rho_{H}\left(\mathbf{u}-\mathbf{u}_{0}\right)=$ 0. Using Lemma 3.2, we immediately obtain an upper bound for the first term:

$$
a\left(\mathbf{u}_{0}, \mathbf{u}_{0}\right) \leq C \eta\left(1+\log \frac{H}{h}\right) a(\mathbf{u}, \mathbf{u}),
$$

where $\eta$ depends on the coefficients. An upper bound for $\eta$ is given by

$$
\max _{1 \leq k \leq K} \max \left(\frac{\gamma_{k}}{\beta_{k}}, \frac{H_{k}^{2} \gamma_{k}}{a_{k}}\right) \text {. }
$$

The upper bound for the remaining terms is established on the subdomain level, and the global result is obtained by summing over all subdomains. For an upper 
bound of $\left\|\mathbf{u}_{i j}\right\|_{0}$, we proceed by further decomposing the subspaces $X_{0}, X_{i j}$, and $X_{k}$ restricted to a substructure into gradient spaces and their orthogonal complements.

We recall that $X_{0}$, restricted to $T_{i}$, is equal to $\mathcal{R}\left(T_{i}\right)$ and thus each $\mathbf{u}_{0} \in X_{0}$ can be written on $T_{i} \in \mathcal{T}_{H}$ as

$$
\left.\mathbf{u}_{0}\right|_{T_{i}}=\operatorname{grad} \phi_{H}+\alpha_{i}\left(\begin{array}{c}
y-y_{i} \\
x_{i}-x
\end{array}\right):=\operatorname{grad} \phi_{H}+\mathbf{u}_{H}
$$

where $\phi_{H}$ is a linear function and $\left(x_{i}, y_{i}\right)$ is the center of gravity of the subdomain $T_{i}$. Then, it can be easily seen that this is a $L^{2}$-orthogonal decomposition and that

$$
\left\|\mathbf{u}_{H}\right\|_{0 ; T_{i}} \leq C H\left\|\operatorname{curl} \mathbf{u}_{H}\right\|_{0 ; T_{i}} .
$$

For the local subspace $X_{i}$, we use the orthogonal splitting already introduced in (15). Each $\mathbf{u}_{i} \in X_{i}$ can be written as

$$
\mathbf{u}_{i}=\operatorname{grad} \phi_{i}+\mathbf{u}_{\perp} .
$$

We denote by $\mathcal{M}(i)$ the set of all indices $1 \leq j \leq K, j \neq i$, such that $T_{i}$ and $T_{j}$ have a common edge and define $\mathbf{u}_{i j}:=\mathbf{u}_{j i}$ in case that $j<i$. By the definition of $X_{i j}$, each $\mathbf{u}_{i j}$ is the gradient of a continuous piecewise linear function $\phi_{i j}$.

By defining

$$
\psi:=\phi_{H}+\phi_{i}+\sum_{j \in \mathcal{M}(i)} \phi_{i j}, \quad \mathbf{w}:=\mathbf{u}_{H}+\mathbf{u}_{\perp}
$$

we obtain the following decomposition for $\mathbf{u}$ on $T_{i}$

$$
\mathbf{u}=\operatorname{grad} \psi+\mathbf{w} .
$$

We remark that this is not an orthogonal decomposition.

It follows, by definition, that $\phi_{H}+\sum_{j \in \mathcal{M}(i)} \phi_{i j}$ is a discrete harmonic function. Applying [23 Lemma 3.3], we obtain

$$
\sum_{j \in \mathcal{M}(i)}\left|\phi_{i j}\right|_{H^{1 / 2}\left(\partial T_{i}\right)}^{2} \leq C\left(1+\log \frac{H}{h}\right)^{2}\left|\phi_{H}+\sum_{j \in \mathcal{M}(i)} \phi_{i j}\right|_{1 ; T_{i}}^{2} .
$$

Using (22), the equivalence between the $H^{1 / 2}$ semi-norm on $\partial T_{i}$ and the $|\cdot|_{1}$ seminorm on $T_{i}$ for discrete harmonic functions, we obtain

$$
\sum_{j \in \mathcal{M}(i)}\left\|\mathbf{u}_{i j}\right\|_{0 ; T_{i}}^{2} \leq C \sum_{j \in \mathcal{M}(i)}\left|\phi_{i j}\right|_{H^{1 / 2}\left(\partial T_{i}\right)}^{2} .
$$

Since $\operatorname{grad} \phi_{i}$ and $\operatorname{grad}\left(\phi_{H}+\sum_{j \in \mathcal{M}(i)} \phi_{i j}\right)$ are orthogonal in $L^{2}\left(T_{i}\right)$, (22) and (23) yield

$$
\sum_{j \in \mathcal{M}(i)}\left\|\mathbf{u}_{i j}\right\|_{0 ; T_{i}}^{2} \leq C\left(1+\log \frac{H}{h}\right)^{2}\|\operatorname{grad} \psi\|_{0 ; T_{i}}^{2} .
$$

In a last step, we have to bound $\|\operatorname{grad} \psi\|_{0 ; T_{i}}^{2}$ by $\|\mathbf{u}\|_{\text {curl } ; T_{i}}^{2}$. Using inequality (17), applied to $\mathbf{u}_{\perp}$, and (20), we obtain

$$
\|\mathbf{w}\|_{0 ; T_{i}}^{2} \leq C_{\perp} H^{2}\left(\left\|\operatorname{curl} \mathbf{u}_{\perp}\right\|_{0 ; T_{i}}^{2}+\left\|\operatorname{curl} \mathbf{u}_{H}\right\|_{0 ; T_{i}}^{2}\right) .
$$

Since curl $\mathbf{u}_{H}$ is constant and curl $\mathbf{u}_{\perp}$ has mean value zero on $T_{i}$, we finally find

$$
\|\mathbf{w}\|_{0 ; T_{i}}^{2} \leq C_{\perp} H^{2}\|\operatorname{curl} \mathbf{w}\|_{0 ; T_{i}}^{2} .
$$


Using (21), we obtain

$$
\|\mathbf{u}\|_{\text {curl } ; T_{i}}^{2}=|\psi|_{1 ; T_{i}}^{2}+\|\mathbf{w}\|_{\text {curl } ; T_{i}}^{2}+2(\mathbf{w}, \operatorname{grad} \psi)_{0 ; T_{i}} .
$$

Applying Young's inequality and (24), we get

$$
\|\mathbf{u}\|_{\text {curl } ; T_{i}}^{2} \geq(1-\epsilon)|\psi|_{1 ; T_{i}}^{2}+\left(1+\left(1-\epsilon^{-1}\right) C_{\perp} H^{2}\right)\|\operatorname{curl} \mathbf{w}\|_{0 ; T_{i}}^{2},
$$

for $0<\epsilon<1$. The choice $\epsilon=C_{\perp} H^{2} /\left(C_{\perp} H^{2}+1\right)$ gives

$$
|\psi|_{1 ; T_{i}}^{2} \leq C\|\mathbf{u}\|_{\text {curl } ; T_{i}}^{2}
$$

and thus

$$
\sum_{j \in \mathcal{M}(i)}\left|\mathbf{u}_{i j}\right|_{0 ; T_{i}}^{2} \leq C\left(1+\log \frac{H}{h}\right)^{2}\|\mathbf{u}\|_{\operatorname{curl} ; T_{i}}^{2}
$$

Summing over all subdomains, we finally get

$$
\left\|\mathbf{u}_{0}\right\|_{\text {curl }}^{2}+\sum_{k=1}^{K}\left\|\mathbf{u}_{k}\right\|_{\text {curl }}^{2}+\sum_{\substack{i, j=1 \\ i<j}}^{K}\left\|\mathbf{u}_{i j}\right\|_{\text {curl }}^{2} \leq C\left(1+\log \left(\frac{H}{h}\right)\right)^{2}\|\mathbf{u}\|_{\text {curl }}^{2} .
$$

Lemma 4.2 is now a consequence of the norm equivalence of the graph norm $\|\cdot\|_{\text {curl }}$ and the energy norm $\|\cdot\|_{a}$.

A detailed analysis of the constant $C$ shows that it depends on the coefficients but not on jumps of the coefficients. It can be bounded by

$$
C \max _{1 \leq k \leq K} \frac{\gamma_{k}}{\beta_{k}}\left(1+\frac{H_{k}^{2} \gamma_{k}}{a_{k}}\right)=: C \chi
$$

where $C$ does not depend any more on the coefficients. In case of time-dependent Maxwell's equations, $\chi$ tends to infinity if the time step tends to zero, and then the constant $C$ in Lemma 4.2 deteriorates. This is due to the interpolation operator $\rho_{H}$ which is not logarithmically stable with respect of the $L^{2}$-norm. In fact the best bound for the $L^{2}$-norm alone is

$$
\left\|\rho_{H} \mathbf{u}\right\|_{0 ; T} \leq C \frac{H}{h}\left\|\rho_{H} \mathbf{u}\right\|_{0 ; T} .
$$

To obtain good results in this important case, we have to modify the decomposition appropriately and find a stable splitting of $\mathbf{u}$ with respect to the $L^{2}$-norm.

We next consider the case where the space $X_{i j}$ in (18) is replaced by the space $\tilde{X}_{i j}$ of discrete Maxwell extensions with respect to the bilinear form $a(\cdot, \cdot)$

$$
\tilde{X}_{i j}:=\left\{\mathbf{v} \in X \mid a(\mathbf{v}, \mathbf{w})=0, \mathbf{w} \in X_{i}, X_{j}, \operatorname{supp} \mathbf{v} \in \bar{T}_{i} \cup \bar{T}_{j}\right\} .
$$

We note that an element $\mathbf{v} \in \tilde{X}_{i j}$ is uniquely defined by its values $\mathbf{n} \times \mathbf{v}$ on $\Gamma_{i j}$. The decomposition

$$
X=X_{0}+\sum_{k=1}^{K} X_{k}+\sum_{\substack{i, j=1 \\ i<j}}^{K} \tilde{X}_{i j}
$$

is now stable with respect to the $L^{2}$-norm as we will show after the proof of the following main theorem. We remark that

$$
X_{0} \subset \sum_{\substack{i, j=1 \\ i<j}}^{K} \tilde{X}_{i j}+\sum_{k=1}^{K} X_{k}
$$


and thus (25), in contrast to (18), is not a direct sum. It follows from Lemma 4.1 that it is sufficient to find one adequate splitting for $\mathbf{u}$.

Theorem 4.3. For each $\mathbf{u} \in X$, there exists a decomposition

$$
\mathbf{u}=\mathbf{u}_{0}+\sum_{k=1}^{K} \mathbf{u}_{k}+\sum_{\substack{i, j=1 \\ i<j}}^{K} \tilde{\mathbf{u}}_{i j}
$$

corresponding to (25) such that

$$
a\left(\mathbf{u}_{0}, \mathbf{u}_{0}\right)+\sum_{k=1}^{K} a\left(\mathbf{u}_{k}, \mathbf{u}_{k}\right)+\sum_{\substack{i, j=1 \\ i<j}}^{K} a\left(\tilde{\mathbf{u}}_{i j}, \tilde{\mathbf{u}}_{i j}\right) \leq C\left(1+\log \left(\frac{H}{h}\right)\right)^{2} a(\mathbf{u}, \mathbf{u})
$$

with a constant $C>0$, independent of $h$ and $\mathbf{u}$.

Proof. The proof is based on the stability of the splitting (18). Each function in $X_{i j}$ can be written as the gradient of a piecewise discrete harmonic function $\phi_{i j}$ with respect to the Laplace operator, but such a representation is not always possible for $\tilde{\mathbf{u}}_{i j} \in \tilde{X}_{i j}$. However, it can be characterized as the solution of a minimization problem. Choosing $\mathbf{u}_{0}=\rho_{H} \mathbf{u}$, which ensures that $\mathbf{u}_{i j} \times\left.\mathbf{n}\right|_{\Gamma_{i j}}=\tilde{\mathbf{u}}_{i j} \times\left.\mathbf{n}\right|_{\Gamma_{i j}}$, we obtain

$$
a\left(\tilde{\mathbf{u}}_{i j}, \tilde{\mathbf{u}}_{i j}\right)=\min _{\substack{\tilde{\mathbf{v}}_{i j} \in X_{0 ; h}\left(T_{i j}\right) \\ \tilde{\mathbf{v}}_{i j} \times \mathbf{n}\left|\Gamma_{i j}=\tilde{\mathbf{u}}_{i j} \times \mathbf{n}\right|_{\Gamma_{i j}}}} a\left(\tilde{\mathbf{v}}_{i j}, \tilde{\mathbf{v}}_{i j}\right) \leq a\left(\mathbf{u}_{i j}, \mathbf{u}_{i j}\right) .
$$

We remark that the coarse space contribution $\mathbf{u}_{0}$ that we have chosen is exactly the same as in the direct space decomposition of Lemma 4.2 .

Finally, we consider the splitting (25) for the limit case $a=0$. In this case, the bilinear form $a(\cdot, \cdot)$ is just a weighted $L^{2}$-scalar product

$$
a(\mathbf{v}, \mathbf{w})=\int_{\Omega} B \mathbf{w} \cdot \mathbf{v} d x
$$

Let us, for the moment, decompose $\mathbf{u}$ as

$$
\mathbf{u}=\sum_{k=1}^{K} \hat{\mathbf{u}}_{k}+\sum_{\substack{i, j=1 \\ i<j}}^{K} \hat{\mathbf{u}}_{i j}
$$

where $\hat{\mathbf{u}}_{i j} \in X$ with $\lambda_{e}\left(\hat{\mathbf{u}}_{i j}\right):=\lambda_{e}\left(\mathbf{u}_{i j}\right), e \subset \Gamma_{i j}$ and $\lambda_{e}\left(\hat{\mathbf{u}}_{i j}\right)=0$ elsewhere, and $\mathbf{u}_{k} \in X_{k}$. Then Lemma 3.1 guarantees that

$$
\sum_{\substack{i, j=1 \\ i<j}}^{K}\left\|\hat{\mathbf{u}}_{i j}\right\|_{0}^{2} \leq C\|\mathbf{u}\|_{0}^{2} .
$$

We remark that $\hat{\mathbf{u}}_{i j}$ is an extension by zero to the interior of the substructures and in general not contained in $\tilde{X}_{i j}$. Taking now the unique decomposition of $\mathbf{u}$ into

$$
\mathbf{u}=\sum_{k=1}^{K} \tilde{\mathbf{u}}_{k}+\sum_{\substack{i, j=1 \\ i<j}}^{K} \tilde{\mathbf{u}}_{i j}
$$


where $\tilde{\mathbf{u}}_{k} \in X_{k}$ and $\tilde{\mathbf{u}}_{i j} \in \tilde{X}_{i j}$, we get, because of the minimization property of $\tilde{\mathbf{u}}_{i j}$,

$$
\sum_{\substack{i, j=1 \\ i<j}}^{K}\left\|\tilde{\mathbf{u}}_{i j}\right\|_{0}^{2} \leq C\|\mathbf{u}\|_{0}^{2} .
$$

This proves the stability of the decomposition of $\mathbf{u}$ with respect to the $L^{2}$-norm. Thus as $\chi$ becomes large, we expect an upper bound for the condition number which is independent of the ratio $H / h$. We remark that this result cannot be obtained with the splitting (18).

Our bound remains valid when the coefficient $B$ tends to zero. In the limit case, $B=0$, the bilinear form $a(\cdot, \cdot)$ is no longer positive definite. However, we can still work with the preconditioned conjugate gradient method in a subspace, if the right-hand side $\mathbf{f}$ is consistent. Then using the stability of $\rho_{H}$ with respect to the $L^{2}$-norm of the curl, (10), we obtain a condition number bound which is independent of $H / h$.

Remark 4.4. We recall that $H(\operatorname{div} ; \Omega)$ is the space of square-summable vectors $\mathbf{u}$ over $\Omega$, with div u square-summable. In two dimensions, any vector in $H$ (div; $\Omega$ ) can be obtained from an element in $H(\operatorname{curl} ; \Omega)$ by a 90-degree rotation; see [10]. Our results and analysis for the space $H(\operatorname{curl} ; \Omega)$ and Nédélec elements are therefore also valid for $H(\operatorname{div} ; \Omega)$ and the lowest-order Raviart-Thomas elements.

Remark 4.5. In the multilevel context, we can immediately get an additive Schwarz method by using a decomposition of $X$ in terms of the hierarchical surplus spaces associated with the different levels and a vertical splitting into curl-free and complementary spaces. Using Lemma 3.2 and a strengthened Cauchy-Schwarz inequality (see [24]) we obtain a method with a condition number that grows quadratically with the number of levels of refinement.

\section{Numerical Results}

In this section, we present some numerical results on the performance of the iterative substructuring method based on the decomposition (25), when varying the diameters of the coarse and fine meshes, and the coefficients $a$ and $B$. We refer to [18, for a general discussion of practical issues concerning Schwarz methods.

We have considered the domain $\Omega=(0,1)^{2}$ and a uniform rectangular triangulations $\mathcal{T}_{h}$ and $\mathcal{T}_{H}$. The fine triangulation $\mathcal{T}_{h}$ consists of $n^{2}$ square elements, with $h=1 / n$. The matrix $B$ is given by

$$
B=\operatorname{diag}\{b, b\} .
$$

In Table1 we show the estimated condition number and the number of iterations in order to obtain a reduction of the residual norm by a factor $10^{-6}$, as a function of the dimensions of the fine and coarse meshes. For a fixed ratio of $H / h$, the condition number is quite insensitive to the fine mesh size. The number of iterations varies slowly with $H / h$ and our results compare well with those for finite element approximations in $H^{1}$ of Laplace's equation; see, e.g., [18]. We remark that the largest eigenvalue is bounded by 5 in all the cases in Table 1 except for $(n=32$, $H / h=16)$ and $(n=64, H / h=32)$; the latter cases correspond to a partition of 2 by 2 subregions and, consequently, the bound for the largest eigenvalue is 3 . 
TABLE 1. Estimated condition number and number of CG iterations (in parentheses) for a residual norm reduction of $10^{-6}$, versus $H / h$ and $n$. Case of $a=1, b=1$.

\begin{tabular}{|l|c|c|c|c|c|}
\hline$H / h$ & 32 & 16 & 8 & 4 & 2 \\
\hline \hline$n=32$ & - & $20.23(11)$ & $26.50(20)$ & $19.10(20)$ & $12.86(17)$ \\
\hline$n=64$ & $26.27(11)$ & $35.94(20)$ & $27.16(21)$ & $19.00(17)$ & $12.90(16)$ \\
\hline$n=128$ & $46.83(20)$ & $36.68(18)$ & $27.06(17)$ & $18.92(16)$ & $*$ \\
\hline$n=192$ & - & $36.71(17)$ & $27.00(17)$ & $18.90(16)$ & $*$ \\
\hline$n=256$ & $47.80(18)$ & $36.66(17)$ & $26.97(16)$ & $18.89(16)$ & $*$ \\
\hline
\end{tabular}

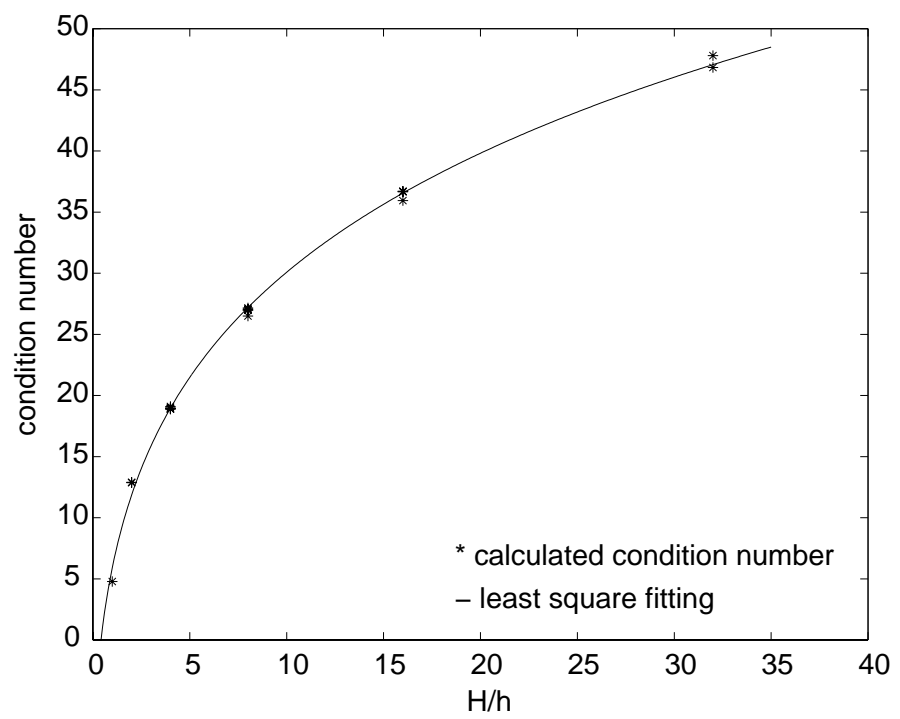

Figure 1. Estimated condition number from Table 1 (asterisk) and least-square second order logarithmic polynomial (solid line), versus $H / h$; relative fitting error about 1.8 percent.

In Figure 1 we plot the results of Table1, together with the best least-square fit second order logarithmic polynomial. The relative fitting error is about 1.8 percent. Our numerical results are therefore in good agreement with the theoretical bound obtained in the previous section and suggest that our bound is sharp.

In Table 2 we show some results when the ratio of the coefficients $b$ and $a$ is changed. The estimated condition number and the number of iterations are shown as functions of $H / h$ and $b$, for a fixed value of $n=128$ and $a=1$. The numerical results also confirm our analysis in the limit cases $b=0$ and $b=\infty$. More precisely, we remark that the condition number tends to be independent of the ratio $H / h$ when the ratio $b / a$ is very small or very large. We recall that when Maxwell's equations are discretized with an implicit time-scheme, the time step is related to the ratio $b / a$. The iterative substructuring method presented in this paper therefore appears very attractive for the solution of linear systems arising from the finite element approximation of time-dependent Maxwell's equations. 
TABLE 2. Estimated condition number and number of CG iterations(in parentheses) for a residual norm reduction of $10^{-6}$, versus $H / h$ and $b$. Case of $n=128$ and $a=1$.

\begin{tabular}{|l|c|c|c|c|}
\hline$H / h$ & 32 & 16 & 8 & 4 \\
\hline \hline$b=1 e-05$ & $3.87(10)$ & $4.68(13)$ & $4.86(13)$ & $4.92(13)$ \\
\hline$b=0.0001$ & $3.87(10)$ & $36.3(16)$ & $26.2(16)$ & $13(15)$ \\
\hline$b=0.001$ & $16.9(11)$ & $36.5(16)$ & $27(16)$ & $18.7(16)$ \\
\hline$b=0.01$ & $46.9(14)$ & $36.7(17)$ & $27.1(16)$ & $18.9(16)$ \\
\hline$b=0.1$ & $46.9(14)$ & $36.7(17)$ & $27.1(17)$ & $18.9(16)$ \\
\hline$b=1$ & $46.8(20)$ & $36.7(18)$ & $27.1(17)$ & $18.9(16)$ \\
\hline$b=10$ & $45.3(22)$ & $36.4(22)$ & $27(18)$ & $18.9(17)$ \\
\hline$b=1 e+02$ & $40.8(25)$ & $34.8(23)$ & $26.7(20)$ & $18.9(19)$ \\
\hline$b=1 e+03$ & $29.8(24)$ & $28.4(23)$ & $24.5(21)$ & $18.4(19)$ \\
\hline$b=1 e+04$ & $17.4(18)$ & $17.3(17)$ & $16.8(18)$ & $15.3(17)$ \\
\hline$b=1 e+05$ & $9.41(14)$ & $9.37(14)$ & $9.3(14)$ & $9.15(14)$ \\
\hline
\end{tabular}

\section{ACKNOWLEDGMENTS}

The authors wish to acknowledge the important role of Dr. Faker Ben Belgacem in the early stages of this project, which began during his visit to the Courant Institute in December 1997.

\section{REFERENCES}

1. Robert A. Adams, Sobolev spaces, Academic Press New York, 1975. MR 56:9247

2. Ana Alonso and Alberto Valli, An optimal domain decomposition preconditioner for lowfrequency time-harmonic Maxwell equations, Math. Comp. 68 (1998), no. 226, 607-631. MR 99i:78002

3. Douglas N. Arnold, Richard S. Falk, and Ragnar Winther, Preconditioning in H(div) and applications, Math. Comp. 66 (1997), 957-984. MR 97i:65177

4. _ Multigrid in H(div) and H(curl), Numer. Math. to appear.

5. - Multigrid preconditioning in $H$ (div) on nonconvex polygons, Comput. Appl. Math. 17 (1998), 303-315. CMP 99:12

6. James H. Bramble, Joseph E. Pasciak, and Alfred H. Schatz, An iterative method for elliptic problems on regions partitioned into substructures, Math. Comp. 46 (1986), no. 173, 361-369. MR 88a:65123

7. Franco Brezzi and Michel Fortin, Mixed and hybrid finite element methods, Springer-Verlag, New York, 1991. MR 92d:65187

8. Robert Dautray and Jaques-Louis Lions, Mathematical analysis and numerical methods for science and technology, Springer-Verlag, New York, 1988. MR 89m:00001

9. Maksymilian Dryja, Barry F. Smith, and Olof B. Widlund, Schwarz analysis of iterative substructuring algorithms for elliptic problems in three dimensions, SIAM J. Numer. Anal. 31 (1994), no. 6, 1662-1694. MR 95m:65211

10. Vivette Girault and Pierre-Arnaud Raviart, Finite element methods for Navier-Stokes equations, Springer-Verlag, New York, 1986. MR 88b:65129

11. Ralf Hiptmair, Multigrid method for $H$ (div) in three dimensions, Electron. Trans. Numer. Anal. 6 (1997), 133-152. MR 99c:65232

12. _ Multigrid method for Maxwell's equations, SIAM J. Numer. Anal. 36 (1998), 204-225. MR 99j:65229

13. Ralf Hiptmair and Andrea Toselli, Overlapping and multilevel Schwarz methods for vector valued elliptic problems in three dimensions, Parallel solution of PDEs, IMA Volumes in Mathematics and its Applications, Springer-Verlag, Berlin, 2000, pp. 181-208. 
14. Jean-Claude Nédélec, Mixed finite elements in $R^{3}$, Numer. Math. 35 (1980), 315-341. MR 81k:65125

15. Charalambos G. Makridakis and Peter Monk, Time-discrete finite element schemes for Maxwell's equations, RAIRO $M^{2} A N 29$ (1995), 171-197. MR 96i:78002

16. Claus Müller, Foundations of the mathematical theory of electromagnetic waves, SpringerVerlag, Berlin, 1969. MR 40:6852

17. Alfio Quarteroni and Alberto Valli, Numerical approximation of partial differential equations, Springer-Verlag, Berlin, 1994. MR 95i:65005

18. Barry F. Smith, Petter E. Bjørstad, and William D. Gropp, Domain decomposition: Parallel multilevel methods for elliptic partial differential equations, Cambridge University Press, 1996. MR 98g:65003

19. Andrea Toselli, Domain decomposition methods for vector field problems, Ph.D. thesis, Courant Institute of Mathematical Sciences, 1999, Technical Report 785, Department of Computer Science, Courant Institute of Mathematical Sciences, New York University.

20. - Overlapping Schwarz methods for Maxwell's equations in three dimensions, Numer. Math. (2000), To appear.

21. _ Neumann-Neumann methods for vector field problems, Tech. Report 786, Department of Computer Science, Courant Institute, June 1999, Submitted to Electron. Trans. Numer. Anal.

22. Andrea Toselli and Axel Klawonn, A FETI domain decomposition method for Maxwell's equations with discontinuous coefficients in two dimensions, Tech. report 788, Department of Computer Science, Courant Institute, September 1999.

23. Olof B. Widlund, Iterative substructuring methods: Algorithms and theory for elliptic problems in the plane, First International Symposium on Domain Decomposition Methods for Partial Differential Equations (Philadelphia, PA) (Roland Glowinski, Gene H. Golub, Gérard A. Meurant, and Jacques Périaux, eds.), SIAM, 1988. MR 90c:65138

24. Barbara I. Wohlmuth, Adaptive Multilevel-Finite-Elemente Methoden zur Lösung elliptischer Randwertprobleme, Ph.D. thesis, TU München, 1995.

25. Barbara I. Wohlmuth, Andrea Toselli, and Olof B. Widlund, Iterative substructuring method for Raviart-Thomas vector fields in three dimensions, SIAM J. Numer. Anal., to appear.

Courant Institute of Mathematical Sciences, 251 Mercer Street, New York, N.Y. 10012

E-mail address: toselli@cims.nyu.edu

URL: http://www.math.nyu.edu/ ${ }^{\sim}$ oselli

Courant Institute of Mathematical Sciences, 251 Mercer Street, New York, N.Y. 10012

E-mail address: widlund@cs.nyu.edu

$U R L:$ http://cs.nyu.edu/cs/faculty/widlund/index.html

Math. Institut, Universität Augsburg, Universitätsstr. 14, D-86 159 Augsburg, GerMANY

E-mail address: wohlmuth@math.uni-augsburg.de

$U R L:$ http://www.hoppe.math.uni-augsburg.de/ ${ }^{\sim}$ wohlmuth 